\title{
Molecular dynamics simulation of peeling a DNA molecule on substrate
}

Received: 18 October 2004 / Revised: 13 January 2005 / Published online: 30 April 2005

(C) Springer-Verlag 2005

\begin{abstract}
Molecular dynamics (MD) simulations are performed to study adhesion and peeling of a short fragment of single strand DNA (ssDNA) molecule from a graphite surface. The critical peel-off force is found to depend on both the peeling angle and the elasticity of ssDNA. For the short ssDNA strand under investigation, we show that the simulation results can be explained by a continuum model of an adhesive elastic band on substrate. The analysis suggests that it is often the peak value, rather than the mean value, of adhesion energy which determines the peeling of a nanoscale material.
\end{abstract}

Keywords Molecular dynamics simulation DNA . Adhesion · Peeling

\section{Introduction}

Recent advances in bioengineering and nanotechnology are opening new frontiers in physical sciences. Functional bionano-hybrid materials which join synthetic nanoscale materials with biological or chemical molecules show enormous promise in combining unique mechanical, electrical, optical and chemical properties of nanostructures with molecular recognition capabilities of biological or chemical species. Various functionalized nanoparticles, nano- tubes and nanowires are finding applications [1-6] in drug delivery, enzyme immobilization, medical diagnostics, molecular probes and sensors, etc. On the other hand, biomolecules can also be used

* The project supported by the Distinguished Young Scholar Fund of NSFC (10225209) and key project from the Chinese Academy of Sciences (KJCX2-SW-L2)

X. Shi $\cdot$ Y. Kong $\cdot$ H. Gao $(\bowtie)$

Max-Planck Institut für Metallforschung,

Heisenbergstr. 3, D-70569 Stuttgart, Germany

E-mail: hjgao@mf.mpg.de

\section{Shi · Y. Zhao}

State Key Laboratory of Nonlinear Mechanics (LNM),

Institute of Mechanics,

Chinese Academy of Sciences, Beijing 100080, China

E-mail: yzhao@lnm.imech.ac.cn as molecular templates to fabricate novel nanostructures [711] for applications in nanoelectronics and nanotechnology. The rapid technological developments are stimulating fundamental understanding, description and regulation of interactions between biomolecules and nanostructures. Mechanics of bio-nano-hybrid materials has the potential of becoming a new research field for the mechanics community to play an important role in guiding the design and manufacturing of nanodevices. In this paper, we perform molecular dynamics simulations to study the adhesion and peeling of a short fragment of single-stranded DNA (ssDNA) oligonucleotide on a solid surface. To simplify the chemical side of the problem, we consider a chemically inert non-polar graphite surface which interacts with the ssDNA primarily via van der Waals forces. A simple analytical model originally developed for peeling of an adhesive elastic band [12-15] from a surface is adopted to describe the peeling of the short ssDNA. This model, while neglecting the entropic contribution to free energy, takes into account the nonlinear elastic property of ssDNA backbone and the non-homogeneous binding energy of different nucleic bases along the ssDNA chain. The predicted peel-off force by this simple model is found to agree quite well with the simulation results.

\section{Methods}

\subsection{Topology and parameters}

The standard topology and parameters for nucleic acids [16] in molecular simulation code CHARMM [17] were used to model ssDNA molecules. For non-bond interactions, the carbon atoms in the graphite substrate were treated as uncharged Lennard-Jones particles with $\sigma_{c c}=3.85 \AA$ and $\varepsilon_{c c}=$ $0.105 \mathrm{kcal} / \mathrm{mol}$ [18]. The covalent carbon-carbon bonds with bond length of $1.42 \AA$ and bond angle of $120^{\circ}$ were maintained by a Morse bond, a harmonic cosine angle, and a cosine torsional potential [18]. The Lennard-Jones parameters for graphite-water interaction were $\sigma_{c o}=3.19 \AA$ and $\varepsilon_{c o}=0.075 \mathrm{kcal} / \mathrm{mol}$ [19], and those for interaction between graphite and nucleic acid atoms were set according 
to a common used combination rules based on geometrical average [20].

\subsection{System setup}

A short homogeneous ssDNA oligonucleotide containing 8 adenine bases in the B-helix form was placed on top of three graphite layers each containing 928 carbon atoms. The hybrid system was solvated in a periodic $90 \times 36 \times 60 \AA$ box with preequilibrated TIP3P water molecules as solvent [21]. Sodium ions were added as counterions to neutralize the negative net charges on the oligonucleotide.

\subsection{Simulation methodology}

The molecular dynamics simulations were performed using CHARMM 28b1 [17]. The SHAKE algorithm [22] was used to fix hydrogen bonds during the simulation. Leapfrog algorithm [23] was employed for integrating the Newton's equation of motion for each atom, with a time step of $1 \mathrm{fs}$. The cutoff for non-bonded interaction was taken to be $12 \AA$, and the non-bonding pair-list was updated every 10 steps. The non-bonded interaction potentials and forces were smoothly shifted to zero at $10 \AA$. In the initial configuration, an ssDNA was placed on graphite with an average separation of about $7 \AA$. The system was equilibrated for $400 \mathrm{ps}$ at $300 \mathrm{~K}$. The temperature was monitored every 100 steps and maintained by scaling velocities only if the deviation of the system average temperature was larger than $10 \mathrm{~K}$. During the simulations, the size of the water box was kept fixed, making it effectively under a constant NVT system. In order to simulate the process of peeling ssDNA from graphite, a dummy atom was linked to one end of the ssDNA with a constraint force constant $K=$ $8 \mathrm{kcal} / \mathrm{mol} / \AA^{2}$. By fixing the graphite and pulling the dummy atom in the designated direction under a constant pulling velocity, steered MD simulations were performed to resemble an AFM experiment [24-29]. The simulations were performed on the equilibrated graphite-oligonucleotide system at different peeling angles. For each peeling angle, multiple pulling rates were selected from $0.01 \AA /$ ps to $0.4 \AA / \mathrm{ps}$.

\subsection{Data processing}

In comparison with MD simulations, the molecules in real AFM experiments are usually pulled at a much lower velocity with much smoother thermal fluctuations. In the simulated peeling, the computed force profiles were processed by Gaussian filter with a half-width of 4 ps. The simulated peeling forces were then extrapolated to a low-velocity limit comparable to the experimental condition.

\section{Results}

\subsection{Adhesion of ssDNA on graphite}

We simulated ssDNA interacting with graphite in water at $300 \mathrm{~K}$. The ssDNA adsorbed on the graphite surface after equilibrium (Fig. 1). All images of the MD results were processed by the VMD software [30]. Since the carbon atoms of graphite are considered to be chemically inert and non-polar Lennard-Jones particles, the vdW attraction is the primary interaction between the ssDNA molecule and the substrate. Figure 2 indicates that the adsorption of ssDNA is mainly due to the vdW interaction between adenine bases and graphite. The planar DNA bases were observed to be parallel to the substrate, with a trend to maximize the contact area, which increases the adhesion energy and stabilizes the ssDNA on substrate. Interestingly, not all nucleic bases were simultaneously attached to the substrate due to the constraint from the ssDNA backbone conformation. With one base attached to the substrate, its nearest-neighbors are forced to stay at a distance away from the substrate. In such a configuration, the work of adhesion between ssDNA and graphite changes according to the relative position of the DNA bases.

The work of adhesion of four DNA bases, adenine, thymine, cytosine and guanine, on the graphite is separately investigated. The simulated adhesion energies, which were listed in Table 1 together with those of deoxyribose (base and sugar ring) on graphite, confirmed that the base-graphite interaction is the primary contribution to the ssDNA-graphite adhesion. For adenine, the simulated adhesion energy of $-21 \mathrm{kcal} / \mathrm{mol}$ is in excellent agreement with previous simulations $(-21 \mathrm{kcal} / \mathrm{mol}[31])$ and experiments $(-23 \mathrm{kcal} / \mathrm{mol}$ [31]). Meanwhile, the simulated adhesion energies of DNA bases in different sites of graphite surface are almost the same; no preferential adsorption configurations on graphite surface were found. This implies that the lateral corrugation barrier of bases on graphite surface is small.

\subsection{Peeling of ssDNA from graphite}

Starting from the equilibrated graphite-oligonucleotide system, steered MD simulations were performed to simulate the peeling of ssDNA from graphite. Figure 3 depicts several snapshots of ssDNA peeled away from graphite at the peeling angle of $\theta=90^{\circ}$. During simulation, one end of the ssDNA oligonucleotide was pulled at a constant velocity of $0.1 \AA / p s$. The computed force profile in Fig. 4 indicated remarkable variations in the pulling force during the simulated peeling process: both the pulling force and the interaction energy reach a maximum value when a tightly attached DNA base is pulled off the surface. On the other hand, the pulling force and the interaction energy decrease to a minimum before the next tightly attached base is to be pulled off. The peel-off force can be calculated from the global maximum of the force profile. The simulations were performed under various pulling rates (from $0.01 \AA / \mathrm{ps}$ to $0.4 \AA / \mathrm{ps}$ ) and peeling angles $\left(75^{\circ}\right.$, $90^{\circ}, 120^{\circ}$ and $135^{\circ}$ ), with the calculated peel-off forces summarized in Fig. 5. The error bar indicates the uncertainties introduced by filtering high-frequency thermal fluctuations in the force profile using Gaussian filters with different half widths. Figure 5 shows that the simulated peel-off forces are strongly dependent on the pulling rate: the higher the rate, the 

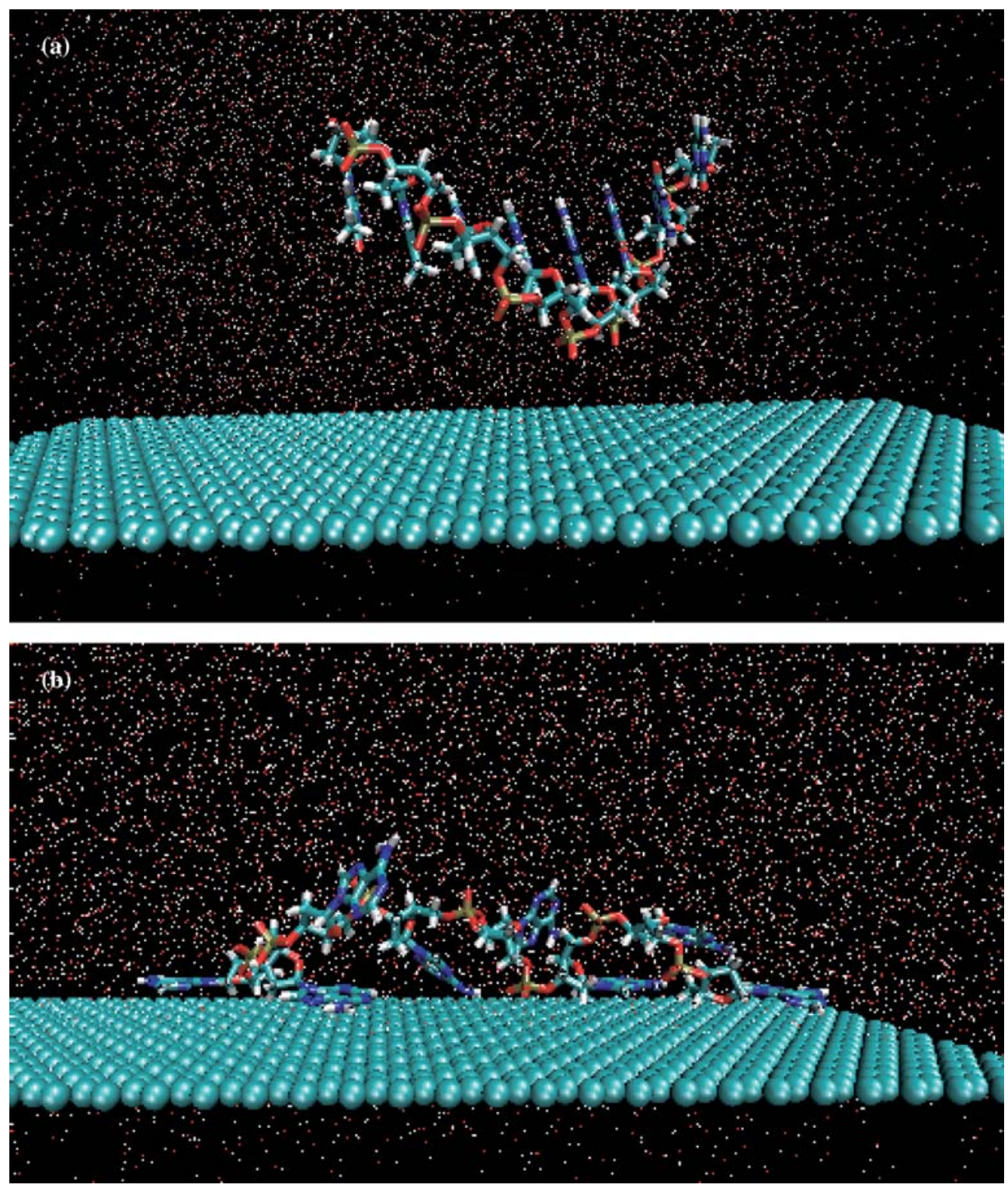

Fig. 1 a,b Adhesion of an ssDNA oligonucleotide on graphite. a The initial and $\mathbf{b}$ the equilibrium conformations

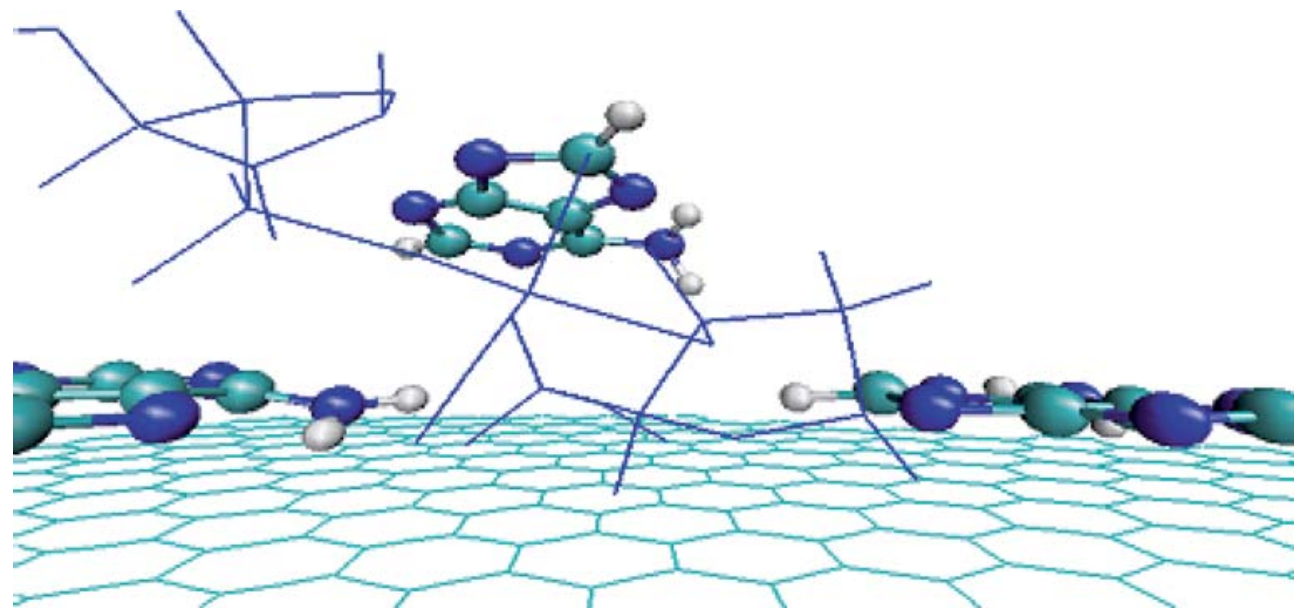

Fig. 2 The non-uniform adsorption profile of ssDNA on graphite leads to variations of adhesion energy along the contour length 


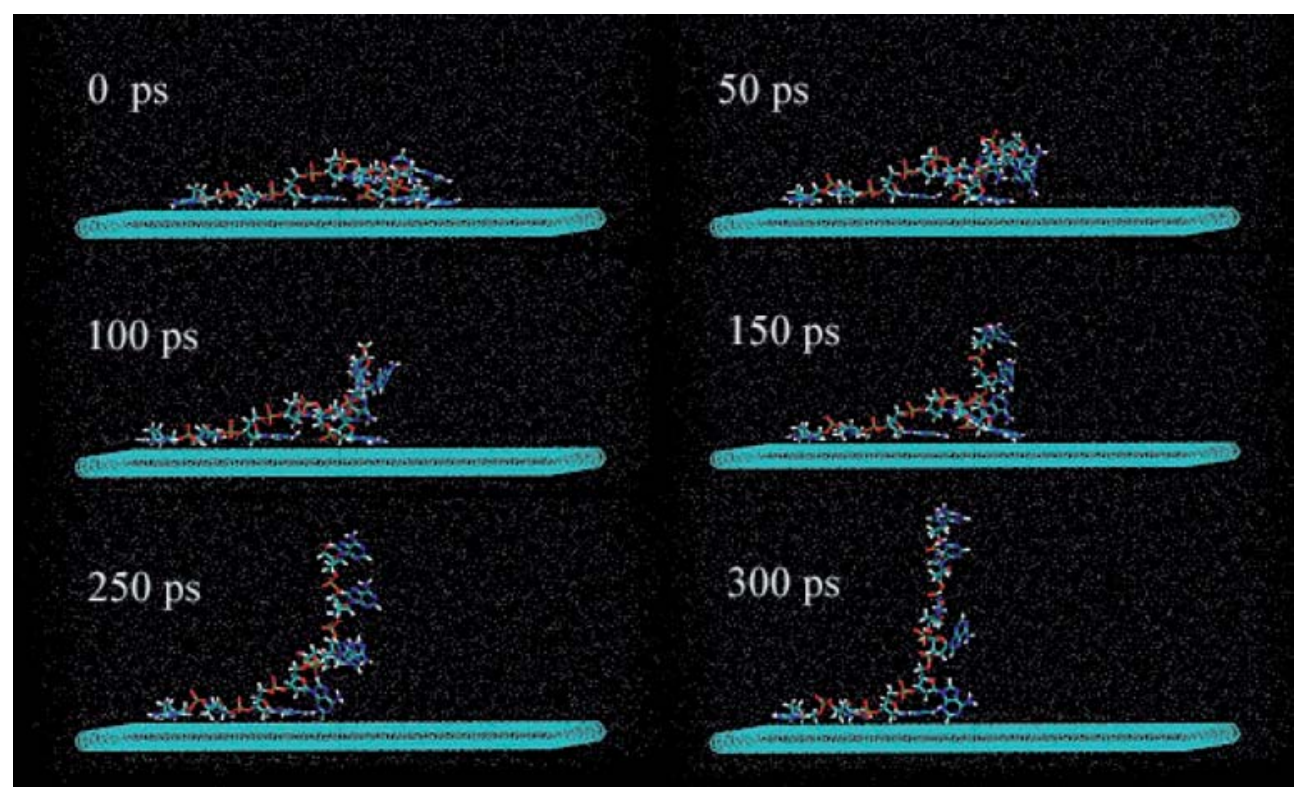

Fig. 3 Steered MD simulation snapshots of peeling ssDNA from graphite at a peeling angle of $90^{\circ}$

Table 1 Simulated adhesion energies of DNA bases with graphite in water by MD. Energy values are given in $\mathrm{kcal} / \mathrm{mol}$

\begin{tabular}{lll}
\hline & Base only & Deoxyribose \\
\hline guanine & -23 & -30 \\
adenine & -21 & -27 \\
thymine & -19 & -26 \\
cytosine & -17 & -24 \\
\hline
\end{tabular}

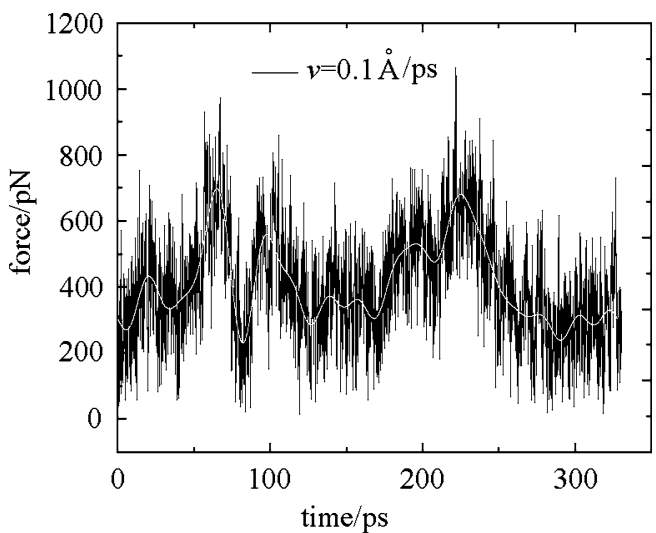

Fig. 4 The pulling force exerted on one end of an adsorbed ssDNA as a function of simulating time. To eliminate excessive thermal noise, the pulling force is smoothed with a Gaussian filter of half width 4 ps (green line)

larger the peel-off forces. Usually, the pulling rate used in MD simulations is several orders of magnitude larger than those in experiments. We thus extrapolated the simulated peel-off forces at multiple pulling rates to the lower limit of the pulling rates to estimate the critical peel-off force for a given peeling angle. The derived critical peel-off forces for the four simulated peeling angles were plotted in Fig. 8.

\section{A generalized elastic band model for peeling of short DNA strands}

Peeling of a thin film or an elastic band from substrate has been traditionally treated by a linear elastic continuum model [12-15]. It has been shown that elasticity of the film tends to reduce adhesion, but the effect of elasticity is significant only for materials capable of supporting very large stresses comparable to elastic modulus or for the case that the peeling angle is very small [12]. Here we apply such a model to interpret the simulated peeling of a short ssDNA. To do this, we will first generalize the elastic band model to a non-linear elastic chain with non-homogeneous adhesion energies.

Consider an adhesive elastic chain subject to a peeling force $\mathbf{F}$ at an angle $\theta$ from a substrate (Fig. 6(a)). Under a virtual infinitesimal decohesion $\delta a$, the energy variation consists of a change of potential energy $F(1-\cos \theta) \delta a$ and a change of elastic energy in the chain. We assume that the elasticity of the polymer obeys a nonlinear law $F=g(\varepsilon)$, where $\varepsilon$ is the elastic strain along the length of the chain. At thermodynamic equilibrium, the critical peel-off force can be determined by the principle of virtual work balance

$F \delta a(1-\cos \theta)+\delta a\left(F \varepsilon-\int_{0}^{\varepsilon} g(\varepsilon) \mathrm{d} \varepsilon\right)=\delta a \Delta \gamma$,

where, $\Delta \gamma$ denotes the work of adhesion between the chain and the substrate. Eliminating $\delta a$ yields

$F(1-\cos \theta)+F \varepsilon-\int_{0}^{\varepsilon} g(\varepsilon) \mathrm{d} \varepsilon=\Delta \gamma$.

This equation is applicable to peeling of a general nonlinear elastic adhesive band on a substrate. When $g(\varepsilon)$ is a linear function, Eq. (2) reduces to a linear elastic peeling model 

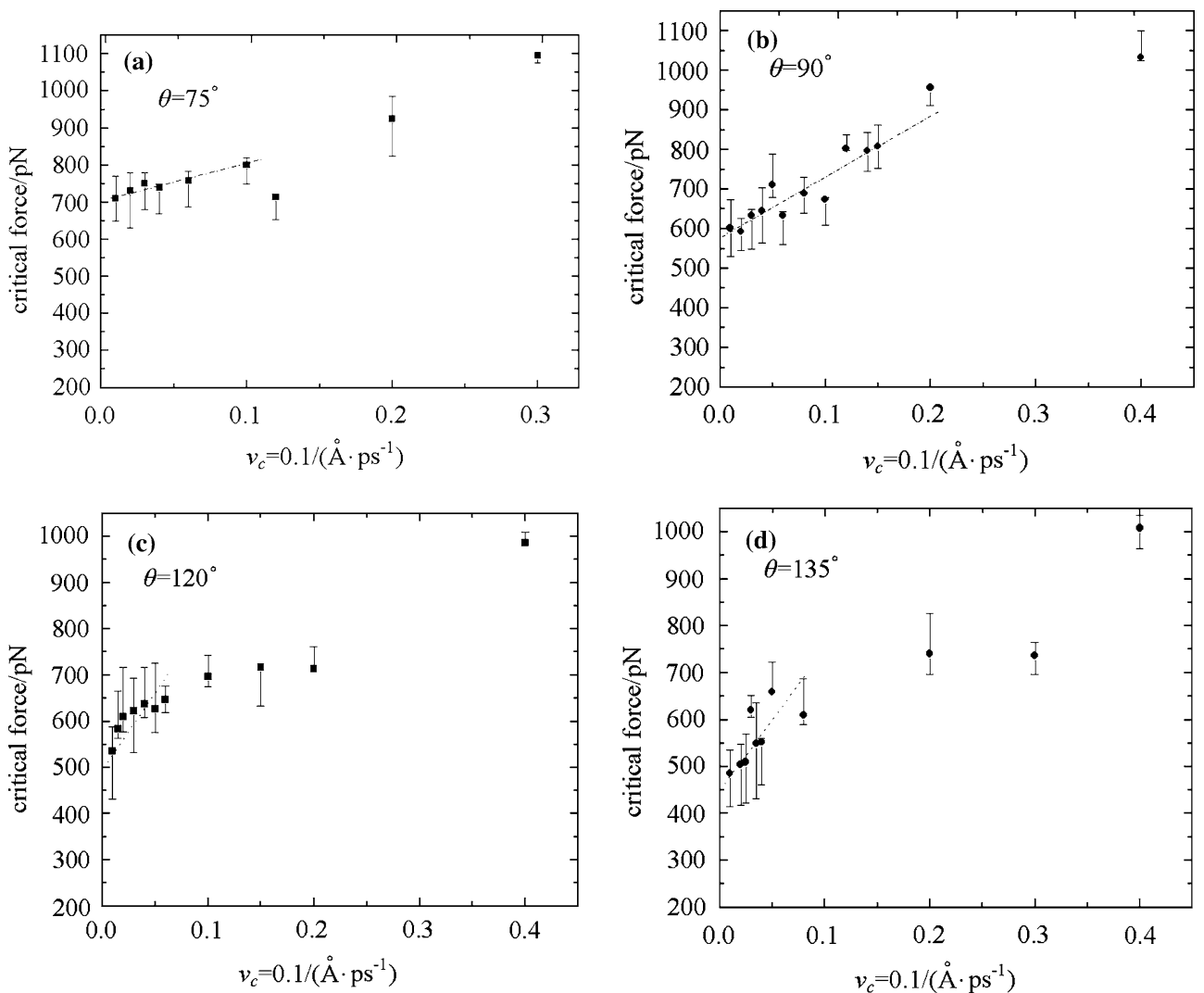

Fig. 5 a-d Simulated peel-off forces as a function of pulling rate at the peeling angles of $\mathbf{a} 75^{\circ}, \mathbf{b} 90^{\circ}, \mathbf{c} 120^{\circ}, \mathbf{d ~} 135^{\circ}$. The error bars show the estimated uncertainty. The dot-dashed line shows a linear extrapolation of the peel-off force to velocities less than $0.01 \AA / p s$

[12-15] used to model the peeling of rubber bands from glass substrates over a range of peeling angles [12]. When the work of adhesion $\Delta \gamma$ is taken as a material constant, the model described by Eq. (2) can be referred to as the homogeneous peeling model.

In order to apply the peeling model of Eq. (2) to the ssDNA adsorbed on graphite, we have determined the elastic function $g(\varepsilon)$ from MD simulations of stretching ssDNA in water. Figure 7 shows the obtained force-strain law $F=g(\varepsilon)$ of ssDNA, which shows consistent trend as experiments [3235]. We used 6-12 Lennard-Jones potential to model van der Waals force between atoms of DNA oligonucleotide and graphite. From the MD simulation of the ssDNA oligonucleotide adsorbed on graphite in water, we estimated the value of $\Delta \gamma$ to be about $5 \mathrm{kcal} / \mathrm{mol}$. $\AA$. Solving Eq. (2) with the estimated $\Delta \gamma$ and $\int_{0}^{\varepsilon} g(\varepsilon) \mathrm{d} \varepsilon$, the critical peel-off force is calculated as a function of the peeling angle and plotted in Fig. 8. The result shows that the homogeneous peeling model predicts correct trend but significantly underestimates the critical peel-off force. The critical peel-off forces obtained from the MD simulations are almost twice of those predicted by the homogeneous peeling model. For example, at the $90^{\circ}$ peeling angle, the model predicts a critical peel-off force of about $322 \mathrm{pN}$ and the MD simulation gives a number of about $600 \mathrm{pN}$.

In the homogeneous peeling model, the work of adhesion between DNA and substrate is treated as a material constant.
However, as the diameter of ssDNA is on the order of $1 \mathrm{~nm}$, the assumption of a constant work of adhesion is no longer accurate. Our simulations indicate that the work of adhesion between ssDNA and graphite changes along the backbone according to the relative positioning of DNA bases. Variations in adhesion energy appear to be a general feature in adhesion and peeling of nanoscale materials from a substrate. To improve the homogeneous peeling model, the homogeneous peeling model is modified as

$$
\begin{gathered}
F(1-\cos \theta)+F \varepsilon-\int_{0}^{\varepsilon} g(\varepsilon) \mathrm{d} \varepsilon \\
=\Delta \gamma^{\prime}\left(1-\left|\sin \frac{\pi}{\ell} x\right|\right),
\end{gathered}
$$

where the constant work of adhesion is replaced by a sinusoidal function with $\ell$ being the period of modulation of adhesion energy and $x$ denoting the position along the chain. In the present case, $\ell$ is the space between two adjacent adhesive peaks. Equation (3) is referred to as the generalized elastic band model (Fig. 6(b)).

For the problem of peeling an ssDNA oligonucleotide from graphite, MD simulations suggested that the spacing between adjacent adhesive peaks should be taken as twice of the spacing $b$ between adjacent nucleic bases, i.e. $\ell=$ $2 b$ (Fig. 2). The position $x=0$ along the backbone corresponds to a base tightly attached to the substrate so that the interaction energy has a maximum value of $\Delta \gamma^{\prime}$. At the 


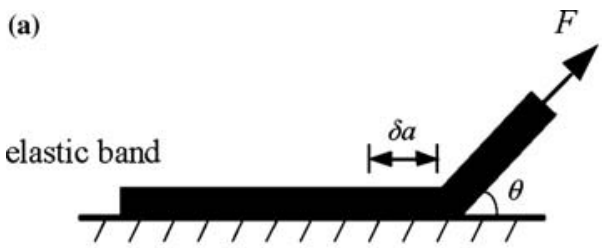

substrate

(b)

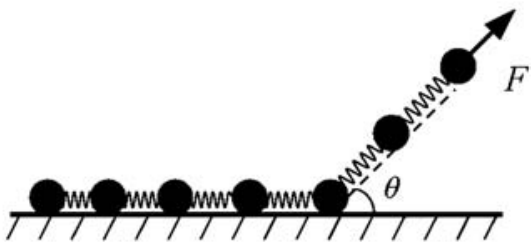

substrate

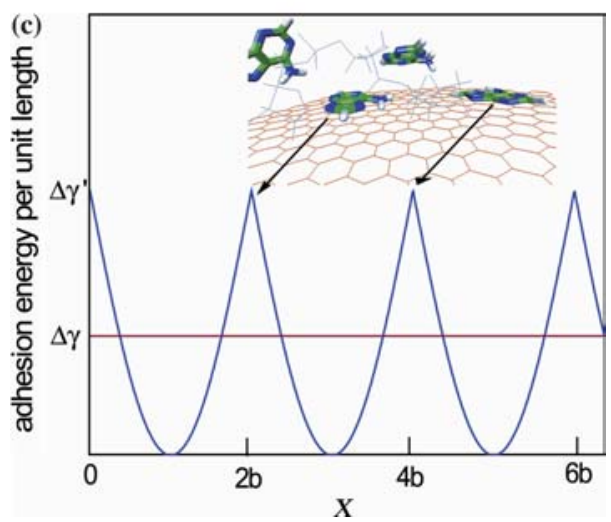

Fig. 6 a Schematic illustration of a homogeneous peeling model of an elastic band on substrate subjected to a peeling force $F$ at angle $\theta$. b The generalized peeling model. c Non-uniform backbone variation of adhesion energy between ssDNA and graphite. In the generalized peeling model, the adhesion energy per unit length is periodically modulated along the backbone, with mean value $\Delta \gamma$ and peak value $\Delta \gamma^{\prime}$

position $x=b$, where the next base remains at a distance away from the substrate, the interaction energy is roughly zero. The non-uniform backbone variation of the interaction energy of ssDNA with substrate is schematically plotted in Fig. 6(c). The value of $\Delta \gamma^{\prime}$ is estimated from the calculated adhesion energies between DNA and graphite in water, which were listed in Table 1 . The value of $\Delta \gamma^{\prime}$ for adenine-graphite interaction was estimated to be about $11 \mathrm{kcal} / \mathrm{mol}$. $\AA$. Solving Eq. (3) yields the critical peel-off forces of ssDNA on graphite according to the generalized elastic band model. The results plotted in Fig. 8 agree well with the simulated values. For instance, at the $90^{\circ}$ peeling angle, the generalized model predicts a critical peel-off force of $622 \mathrm{pN}$, and the MD result is $600 \mathrm{pN}$. This analysis indicated that it is the peak value, rather than the mean value, of adhesion energy along the backbone which determines the critical peel-off force of ssDNA. The same conclusion could be expected for nanoscale peeling in general.

How important is the effect of elasticity in peeling of ssDNA? To answer this question, we have recalculated the critical peel-off force of ssDNA on graphite without the elastic

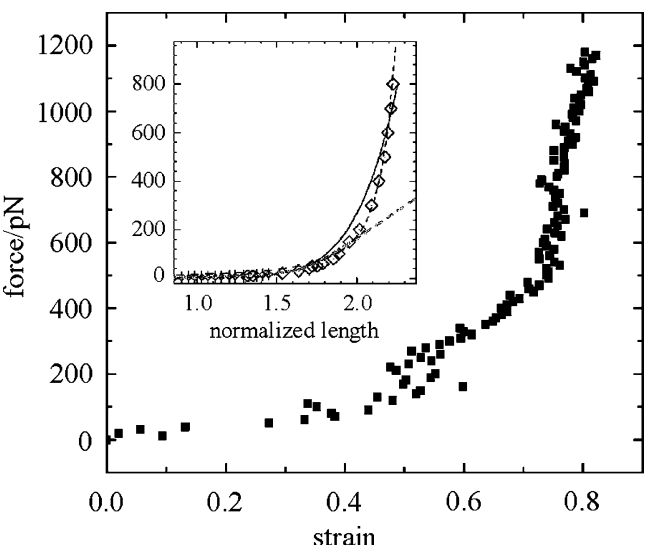

Fig. 7 The simulated stretching force of ssDNA as a function of strain. An 8-base segment of ssDNA is stretched in water at room temperature. Inset is experimental data where extension of ssDNA has been normalized by the contour length of an equivalent ssDNA molecule $[35,36]$

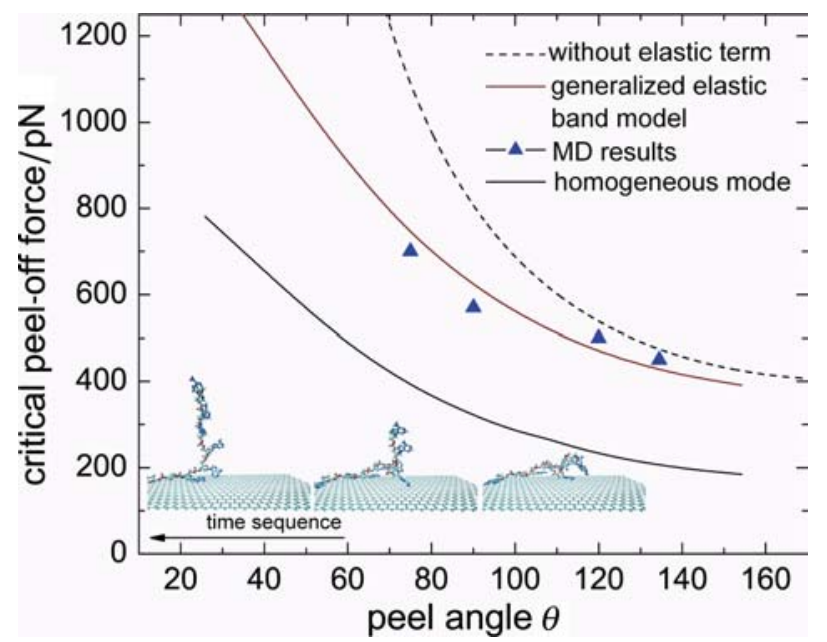

Fig. 8 (a) The critical peel-off forces of an ssDNA oligonucleotide on graphite as a function of the peeling angle. The black line shows the prediction of the homogeneous peeling model, the red line that of the generalized elastic band model and the blue closed triangles are the results of direct $\mathrm{MD}$ simulations at peeling angles of $75^{\circ}, 90^{\circ}, 120^{\circ}$, and $135^{\circ}$. The dashed line shows the predictions of the generalized peeling model without accounting for the effect of elasticity

term in Eq. (3). The results, plotted in dashed line in Fig. 8, showed that the elasticity of ssDNA plays an important role, with increasing effect as the peeling angle decreases. Whereas the difference in calculated critical peel-off force is negligible for the peeling angles larger than $120^{\circ}$, the peel-off force calculated for smaller angles without the elastic term is significantly larger than those of the MD simulation (Fig. 8).

\section{Discussion}

The simulated critical peel-off forces of ssDNA on graphite showed strong dependence upon the peeling angle. In an earlier study of cell-cell adhesion, Bell [36] pointed out that the 
strength of biomolecular bonds should depend on the slope of the free energy along the unbinding path rather than on the free energy difference. In the case of adhesion of a short ssDNA fragment on substrate, our simulations and models demonstrated that, for the same adhesion energy, the critical peel-off forces also depend on the peeling angle and elasticity.

A disadvantage of our elastic peeling model is that the effect of entropy is not explicitly considered. For the short ssDNA fragment under investigation, the entropic contribution to elasticity can be considered lumped into the nonlinear elastic function $g(\varepsilon)$ in Eqs. (1)-(3). This simplification is expected to break down as the contour length of the chain increases. In general, statistical mechanics approaches must be adopted to describe the behavior of polymeric chains. Further discussion in this respect is beyond the scope of this work.

\section{Conclusion}

In conclusion, we have used molecular dynamics simulations to study the adsorption and peeling of a short fragment of ssDNA molecule on a graphite surface. The simulated critical peel-off forces of ssDNA depend on both the peeling angle and the elasticity of the chain. We found that the simulation results can be explained by a generalized elastic band model accounting for both the nonlinear elasticity and the non-uniform adhesion energy along the length of ssDNA. The calculated critical peel-off force for different peeling angles agrees well with the MD simulation results.

Acknowledgements The work of XS was supported by a Max Planck Graduate Fellowship during 2002-2003. HG acknowledges support from the Max Planck Society, the NSFC and the Chang Jiang Scholarship of Tsinghua University.

\section{References}

1. Bruchez, M, Moronne, M, Gin, P, Weiss, S, Alivisatos, AP: Semiconductor nanocrystals as fluorescent biological labels. Science, 281: 2013-2016 (1998)

2. Chan, W, Nie, S: Quantum dot bioconjugates for ultrasensitive nonisotopic detection. Science, 281: 2016-2018 (1998)

3. Wong, SS, Joselevich, E, Woolley, AT, Cheung, CL, Lieber, CM: Covalently functionalised nanotubes as nanometre-sized probes in chemistry and biology. Nature, 394: 52-55 (1998)

4. Taton, T, Mirkin, C, Letsinger, R: Scanometric DNA array detection with nanoparticle probes. Science, 289: 1757-1760 (2000)

5. Cui, Y, Wei, Q, Park, H, Lieber, CM: Nanowire nanosensors for highly sensitive and selective detection of biological and chemical species. Science, 293: 1289-1292 (2001)

6. Qi, P, Vermesh, O, Grecu, M, Javey, A, Wang, Q, Dai, H: Toward large arrays of multiplex functionalized carbon nanotube sensors for highly sensitive and selective molecular detection. Nano Lett, 3: 347-351 (2003)

7. Miemeyer, CM: Nanoparticles, proteins, and nucleic acids: biotechnology meets materials science. Angew Chem Int Ed, 40: 4128-4158 (2001)

8. Martin, CR, Kohli, P: The emerging field of nanotube biotechnology. Nature Reviews Drug Discovery, 2: 29-37 (2003)
9. Bashir, R: DNA-mediated artificial nanobiostructures: state of the art and future directions. Superlattice and Microstructures, 29: 1$16(2001)$

10. Xin, H, Woolley, AT: DNA-templated nanotube localization. J Am Chem Soc, 125: 8710-8711 (2003)

11. Reches, M, Gazit, E: Casting metal nanowires within discrete selfassembled peptide nanotubes. Science, 300: 625-627 (2003)

12. Kendall, K: Thin-film peeling the elastic term. J Phys D: Appl Phys, 8: $1449-1452$ (1975)

13. Kendall, K: The adhesion and surface energy of elastic solids. J Phys D: Appl Phys, 4: 1186-1195 (1971)

14. Kendall, K: Transition between cohesive and interfacial failure in a laminate. Proc R Soc A, 344: 287-302 (1975)

15. Lindley, PB: Ozone attack at a rubber-metal bond. J Instn Rubb Ind, 5(6): 243-248 (1971)

16. MacKerell, Jr AD, Wi-rkiewicz-Kuczera, J, Karplus, M: An allatom empirical energy function for the simulation of nucleic acids. J Am Chem Soc, 117: 11946-11975 (1995)

17. Brooks, BR, Bruccoleri, RE, Olafson, BD, States, DJ, Swaminathan, S, Karplus, M: CHARMM: a program for macromolecular energy, minimization and dynamics calculations. J Comp Chem, 4: 187-217 (1983)

18. Rappé, AK, Casewit, CJ, Colwell, KS, Goddard, III WA, Skiff, WM: UFF, a full periodic table force field for molecular mechanics and molecular dynamics simulations. J Am Chem Soc, 114 10024-10035 (1992)

19. Walther, JH, Jaffe, R, Halicioglu, Koumoutsakos, P: Carbon nanotubes in water: structural characteristics and energetics. J Phys Chem B, 105: 9980-9987 (2001)

20. van der Spoel, D et al.: GROMACS user manual, version 3.1.1. Groningen, The Netherlands.

21. Jorgensen, WL, Chandrasekhar, J, Madura, JD, Impey, RW, Klein, ML: Comparison of simple potential functions for simulating liquid water. J Chem Phys, 79: 926-935 (1983)

22. Ryckaert, JP, Cicotti, G, Berensden, HJC: Numerical integration of the cartesian equations of motion of a system with constraints: molecular dynamics of n-alkanes. J Comp Phys, 23: 327-341 (1977)

23. Honeycutt, RW: The potential calculation and some applications. Methods in Computational Physics, 9: 136-211 (1970)

24. Grubmüller, H, Heymann, B, Tavan, P: Ligand binding: molecular mechanics calculation of the streptavidin-biotin rupture force. Science, 271: 997-999 (1996)

25. Heymann, B, Grubmüller, H: AN02/DNP-hapten unbinding forces studied by molecular dynamics atomic force microscopy simulations. Chem Phys Lett, 303: 1-9 (1999)

26. Izrailev, S, Stepaniants, S, Balsera, M, Ono, Y, Schulten, K: Molecular dynamics study of unbinding of the avidin-biotin complex. Biophys J, 72: 1568-1581 (1997)

27. Marrink, SJ, Berger, O, Tieleman, P, Jähnig, F: Adhesion forces of lipids in a phospholipid membrand studied by molecular dynamics simulations. Biophys J, 74: 931-943 (1998)

28. Evans, E, Ritchrie, K: Dynamic strength of molecular adhesion bonds. Biophys J, 72: 1541-1555 (1997)

29. Merkel, R, Nassoy, P, Leung, A, Ritchrie, K, Evans, E: Energy landscapes of receptor-ligand bonds explored with dynamic force spectroscopy. Nature, 397: 50-53 (1999)

30. Humphrey, W, Dalke, A, Schulten, K: VMD-visual molecular dynamics. J Molecular Graphics, 14: 33-38 (1996)

31. Edelwirth, M, Freund, J, Sowerby, SJ, Heckl, WM: Molecular mechanics study of hydrogen bonded self-assembled adenine monolayers on graphite. Surf. Sci, 417: 201-209 (1998)

32. Smith, S, Cui, Y, Bustamante, C: Overstretching B-DNA: the elastic response of individual double-stranded and single-stranded DNA molecules. Science, 271: 795-799 (1996)

33. Wuite, G, Smith, S, Young, M, Keller, D, Bustamante, C: Single-molecule studies of the effect of template tension on T7 DNA polymerase activity. Nature, 404: 103-106 (2000)

34. Maier, B, Bensimon, D, Croquette, V: Replication by a single DNA polymerase of a stretched single-stranded DNA. Proc Natl Acad Sci USA, 97: 12002-12007 (2000) 
35. Dessinges, MN, Maier, B, Peliti, M, Bensimon, D, Croquette, V: Stretching single stranded DNA, a model polyelectrolyte. Phys Rev Lett, 89: 248102 (2002)
36. Bell, G: Models for the specific adhesion of cells to cells. Science, 200: 618-627 (1978) 